EPJ Web of Conferences 61, 07001 (2013)

DOI: 10.1051/epjconf/20136107001

(C) Owned by the authors, published by EDP Sciences, 2013

\title{
Multi-frequency Polarization Studies of AGN Jets
}

\author{
Denise C. Gabuzda1,a \\ ${ }^{1}$ Department of Physics, University College Cork, Cork, Ireland
}

\begin{abstract}
Throughout most of the history of radio interferometry studies of Active Galactic Nuclei (AGN) using VLBI, attempts to extract physically meaningful information from VLBI observations have been dominated by visibility-based approaches to analysis, such as model fitting of the self-calibrated visibility data. This approach has been extremely valuable in multi-epoch studies of superluminal motion, for example; however, as polarization-sensitive and multi-frequency studies of the parsec-scale jets of AGN have become more common, it has often proven difficult to unambiguously relate features observed in total intensity and polarization, or at multiple frequencies. This has necessitated the development of new image-based analysis techniques, as well as studies aimed at improving our understanding of the uncertainties in quantities derived from images. These new techniques have provided a wide range of key information about the relativisitic jets of AGN on parsec scales, including measurements of typical magnetic-field strengths in the VLBI core components and information about the three-dimensional magnetic-field structures of the jets. These properties of the jets are, in turn, intrinsically related to fundamental questions such as the formation, launching and collimation of the jet structures.
\end{abstract}

\section{Introduction}

One of the most exciting discoveries in the early days of Very Long Baseline Interferometry (VLBI) studies of Active Galactic Nuclei (AGN) jets was the detection of superluminal motion in the quasars 3C279 and 3C273 [8]. This was based on observations with a very limited VLBI array, and was detectable only through the measurement of small changes in the visibility functions. As images became available, the observation of individual "components" that seemed to move with superluminal speeds made it clear that this phenomenon could provide key information about the relativistic jets of AGN. This essentially led to a dominance of visibility-based analyses of VLBI data in the 1970s-1990s, in particular, model-fitting of total-intensity visibility functions using circular or elliptical Gaussian components or optically thin spheres. Attempts were also made to model-fit the linearly polarized visibility data, but it was fairly often not possible to unambiguously identify features in total intensity and polarization. Attempts to model-fit polarization data are also plagued by the need to model fit data for the Stokes parameters $Q$ and $U$ separately, when, again, it was not always clear how features in the $Q$ and $U$ fits should be cross-identified. All this makes it difficult in some cases to derive the degree of polarization and the orientation of the polarization electric vector relative to the local jet flow for some polarized regions through model-fitting. Similarly, multi-frequency observations sometimes yielded a good coincidence between the jet structures observed at different frequencies,

\footnotetext{
ae-mail: d.gabuzda@ucc.ie
}

but also frequently revealed features that were prominent at some frequencies and didn't have clear counterparts at others.

Since the beginning of the operation of the Very Long Baseline Array (VLBA) in the early 1990's and the accompanying software developments, image-based approaches to the analysis of VLBI data have been becoming the norm. Continuous distributions of the degree of polarization, spectral index, and Faraday rotation measure are increasingly considered, sometimes in parallel with visibility model-fitting. Two key types of image-based studies that are discussed below are estimates of core magnetic (B) field strengths based on measurement of the frequency-dependent shift of the VLBI core position (Section 2) and Faraday-rotation studies of both the ambient medium through which AGN jets propagate and the threedimensional B-field structures of these jets (Section 3 ).

Pixel-based analyses of VLBI images have usually either ignored the question of the uncertainties in quantities measured in individual image pixels, or assumed that the uncertainty in an intensity measured within a single pixel is equal to the root-mean-square (rms) deviation of the flux values about their mean in parts of the image far from source emission. This assumption seemed plausible, and was widely applied without any firm justification for roughly two decades, until a number of recent studies carried out in the past few years, which will be discussed in Section 4. 


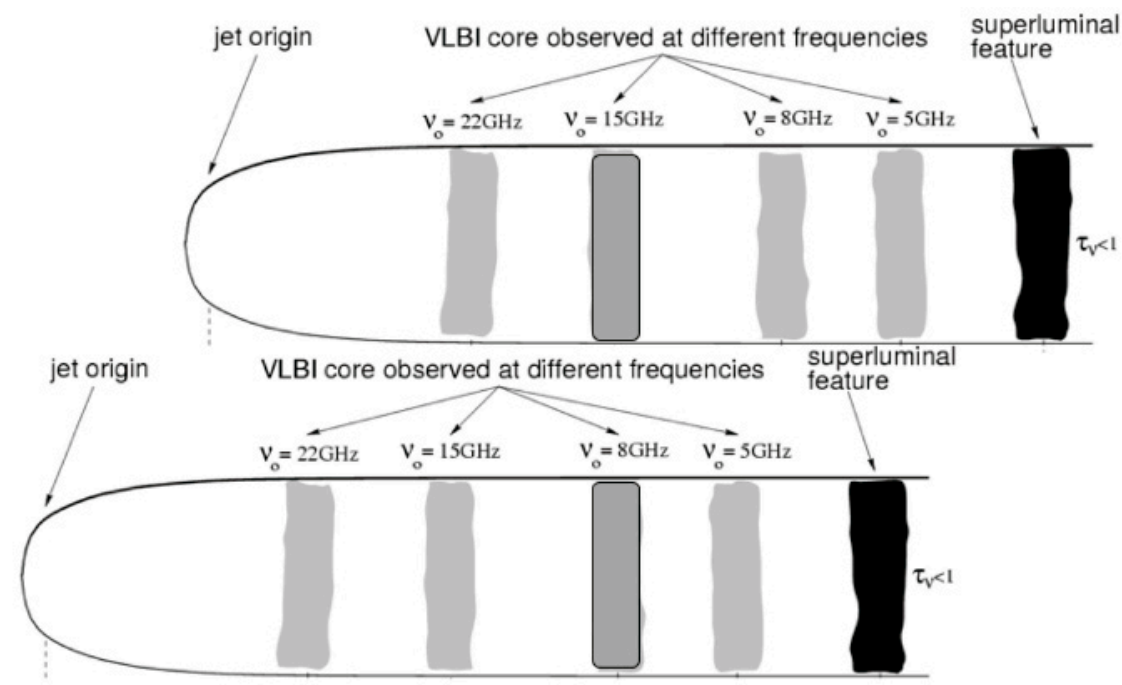

Figure 1. Schematic illustrating why VLBI images made at different frequencies will be misaligned. Considered here is the example of comparing observations at $15 \mathrm{GHz}$ (top "jet") and $8 \mathrm{GHz}$ (bottom "jet"). The correct physical alignment is such that the black optically thin superluminal features are coincident, but the mapping process aligns the optically thick core regions at the two frequencies, represented by the dark grey feature outlined in black. Based on a figure from [19].

\section{Image Alignment, Core Shifts and Core B Fields}

In the model of Blandford \& Königl [5], the compact feature observed as the "VLBI core" represents the optically thick base of the jet, roughly corresponding to the surface in the jet where the optical depth $\tau \approx 1$. The location of this surface depends on the observing frequency, and moves further down the jet at increasingly lower frequencies, as is shown in the schematic in Fig. 1, based on a figure from Kovalev et al. [19]. In contrast, we expect the positions of optically thin features in the jet to coincide at different frequencies. However, absolute position information is lost during the self-calibration and mapping process, and the phase center of a VLBI image will tend to coincide with the dominant, partially optically thick VLBI core. Therefore, VLBI images obtained at different frequencies will not be correctly aligned in a physical sense, and direct superposition of such images will yield erroneous spectral-index maps. To correctly align images obtained at different frequencies, it is necessary to shift them so that the positions of optically thin features coincide at the different frequencies.

A variety of techniques for achieving this alignment have been exploited, most importantly model-fitting in the visibility domain to determine the positions of optically thin jet features, which are then made to coincide at different frequencies, and cross-correlation of the optically thin parts of total intensity images (e.g. $[11,35])$. In practice, different approaches may work best for different types of soruce structures, and both of these general approaches can yield reliable alignments in particular cases.

The first study aimed at using the frequency dependence of AGN VLBI-core positions to estimate the core B-

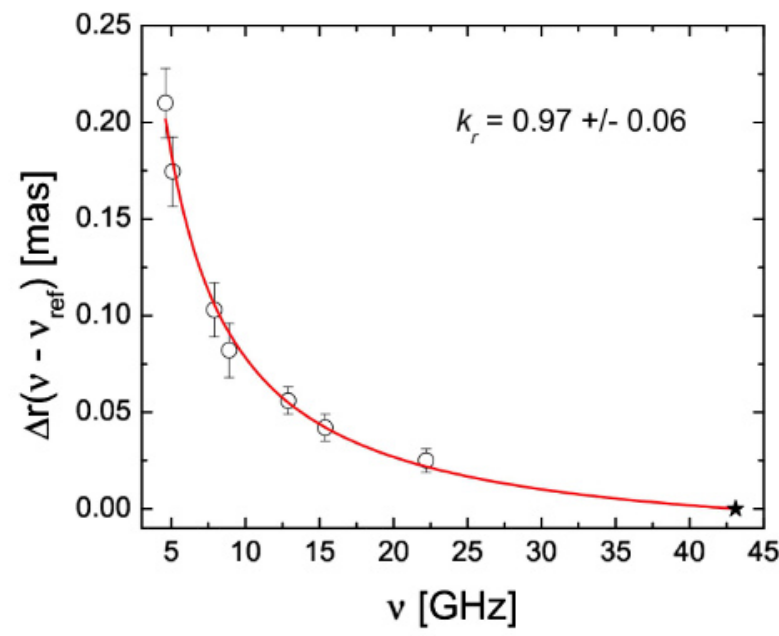

Figure 2. Plot of derived position of the VLBI core relative to the position at the reference frequency of $43 \mathrm{GHz}$ as a function of frequency, showing the power-law behaviour expected for a Blandford-Königl jet; the implied value of the parameter $k_{r}$ is equal to unity within the errors, consistent with the core region being in equipartition. Taken from O'Sullivan \& Gabuzda [29].

field strengths was carried out by Lobanov [22]. Based on images at four frequencies, this analysis showed that the position of the VLBI core seemed to show the expected sort of frequency dependence, but only limited conclusions could be drawn, due to the limited number of frequencies and sources considered. Nevertheless, this work was important, as it laid out an approach to estimating the core B fields based on measurement of the core position as a function of frequency. 


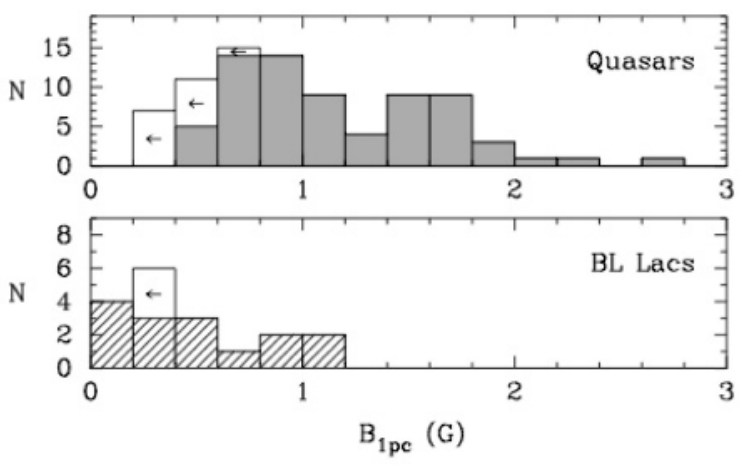

Figure 3. Histograms of the derived B fields of quasars and BL Lac objects at a distance of $1 \mathrm{pc}$ from the jet base. Taken from Pushkarev et al. [30].

After an interval of more than a decade, there has recently been a burst of new core-shift studies, most notably those of Kovalev et al. [19], O’Sullivan \& Gabuzda [29], Sokolovsky et al. [31] and Pushkarev et al. [30]. O'Sullivan \& Gabuzda [29] were the first to carry out a core-shift analysis for VLBI observations obtained simultaneously at more than four frequencies. Their data based on eight frequencies from 4.6 to $43 \mathrm{GHz}$ provided detailed and redundant measurements, which clearly demonstrated the expected power-law dependence of the core position as a function of frequency (Fig. 2). These measurements also provided evidence that the parameter $k_{r} \simeq 1$ in most of the six AGN considered, where the position of the VLBI core $r$ as a function of frequency $v$ is given by $r \propto v^{-1 / k_{r}}$; a reasonable interpretation of the result that $k_{r} \simeq 1$ is that these core regions are close to equipartition, with the magnetic field $B \sim r^{-1}$ and the electron density $N \sim r^{-2}$. This is potentially a very important result, since equipartition has often been assumed for various calculations, but without any observational evidence that this assumption is reasonable. Kovalev et al. [19] were the first to derive coreshift measurements for a much larger number of sources (29), but at only two frequencies, while Sokolovsky et al. [31] obtained results for 20 sources at nine frequencies. Most recently, Pushkarev et al. [30] obtained core-shift data for more than a hundred well-studied sources from the MOJAVE project [21] at four frequencies from 8.1 to 15.4 GHz. The core-region B fields inferred from all these studies are generally a few tenths of a Gauss. Pushkarev et al. [30] found evidence that the core B fields of quasars are somewhat higher than those in BL Lac objects (Fig. 3); this is presumably related to the higher luminosities of quasars in some way, but exactly how is not entirely clear.

\section{Faraday Rotation Studies of the Ambient Medium and Jet B Fields}

Faraday rotation is a rotation of the observed linear polarisation angle $\chi$ when a polarised electromagnetic wave passes through a magnetised plasma (containing free electrons and magnetic field); this comes about due to the different indices of refraction of the plasma for the rightcircularly-polarized and left-circularly-polarized components of the wave. The amount of rotation is given by $\operatorname{RM} \lambda^{2}$, where $\lambda$ is the observing wavelength and

$$
R M=\frac{e^{3}}{8 \pi^{2} \epsilon_{o} m_{e}^{2} c^{3}} \int n_{e} \mathbf{B} \cdot d \mathbf{l}
$$

where $e$ and $m_{e}$ are the charge and mass of the Faradayrotating charges, $n_{e}$ is the charge density in the region of Faraday rotation and the integral is carried out over the line of sight from the source to the observer. Due to the inverse dependence on the square of the mass of the Faradayrotating charges, it is usually assumed that observed Faraday rotation is due to the action of thermal electrons. The magnitude of the rotation measure RM depends on both $n_{e}$ and the line-of-sight component of the magnetic field, while the sign of the Faraday rotation is determined by the direction of the line-of-sight magnetic field (either toward or away from the observer).

A number of early Faraday-rotation studies of AGN on parsec scales were carried out by Taylor [32, 33] and Zavala \& Taylor [36, 37], who were primarily interested in looking for differences between the core and jet RM values. These Faraday-rotation maps for about 40 AGN showed a clear tendency for the core RMs to be greater in magnitude than the jet RMs, apparently due to higher electron densities and magnetic-field strengths on smaller scales in the jets. These studies also presented evidence that the core RMs of quasars tend to be higher than those of BL Lac objects; it is an obvious possibility that this is related to the stronger optical line emission of quasars, compared to BL Lac objects, but details of this sort of picture are unclear.

\subsection{Faraday Rotation and Helical Jet B Fields}

As was pointed out by Blandford [4], if a jet has a helical B field, we should observe a Faraday-rotation gradient across the jet, due to the systematically changing line-ofsight component of the B field across the jet. Thus, the detection of transverse Faraday-rotation gradients across AGN jets could potentially provide a powerful diagnostic for the presence of an azimuthal field component associated with a helical or toroidal jet B field. The theoretical simulations of Broderick \& McKinney [6] directly demonstrate the development of helical jet $B$ fields and the resulting presence of Faraday rotation gradients across the simulated jets.

A number of transverse Faraday-rotation gradients across AGN jets have been reported in the literature, most recently by Asada et al. [3], Croke et al. [12], Gómez et al. [17], Hovatta et al. [18], Mahmud et al. [26] and Gabuzda 


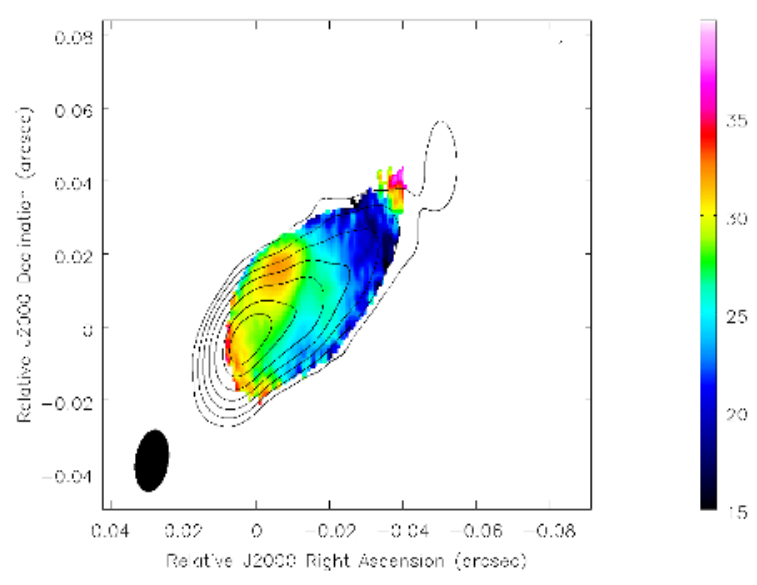

Figure 4. Faraday RM map of 3C380 produced by Gabuzda et al. [15], based on VLBA data at 1.4, 1.6 and $5 \mathrm{GHz}$. This image clearly shows an RM gradient across the jet, whose significance is about $4 \sigma$.

et al. [15] [see Fig. 4]. Gómez et al. [17] superposed degree of polarization and RM images for 3C120 obtained at multiple epochs to effectively map out these quantities along a much longer portion of the jet than was possible at any single epoch, making the transverse structure in the polarization and Faraday rotation appreciably clearer.

Although transverse RM gradients could, in principle, come about due to gradients in the electron density in some cases, transverse RM gradients reliably exhibiting a sign change cannot be due to electron-density gradients; therefore, reliable transverse RM gradients encompassing RMs of both signs provide particularly strong evidence for helical or toroidal jet B fields. Such B fields could come about in a natural way as a consequence of the rotation of the central black hole and its accretion disk, together with the jet outflow.

An important question is whether the azimuthal field component revealed by a transverse RM gradient is associated with a helical or a toroidal B field. Generally speaking, it is only asymmetry of the intensity and polarization profiles across the jet that can distinguish observationally between a helical field (with an ordered poloidal field component) and a toroidal field (with a disordered poloidal field component). Here, we must recall that the degree of polarization is determined by the component of the jet magnetic field in the plane of the sky (e.g., a magnetic field directed precisely toward the observer would give no linear polarization). Fig. 5 shows qualitatively that the poloidal component of a helical field that is not viewed at $90^{\circ}$ in the rest frame of the jet (i.e., viewed "from the side") makes the overall field lie predominantly along the line of sight at one edge of the helix (giving rise to higher Faraday rotation and lower degrees of polarization), and predominantly in the plane of the sky at the other edge of the helix (giving rise to lower Faraday rotation and higher degrees of polarizaton). In contrast, in the absence of other factors, a toroidal field should give rise to a symmetric po-

\section{B close to Higher Faraday rotation, \\ line of sight lower degree of polarization

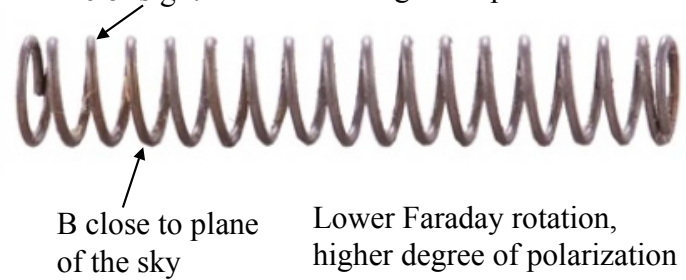

Figure 5. Schematic illustrating the connection between asymmetry in the polarization and RM profiles across a jet and the presence of an ordered poloidal field component associated with a helical field. A purely toroidal field will give rise to symmetric profiles, independent of the viewing angle.

larization profile across the jet, independent of viewing angle.

Murphy et al. [27] have carried out fitting of the transverse total intensity and polarization profile across the jet of Mrk501 on parsec scales; these polarization profiles display appreciable asymmetry, suggestive of a helical rather than a toroidal B field, and are, in fact, fit well using a simple helical-field model with a pitch angle of about $53^{\circ}$ and a viewing angle in the jet rest frame of about $83^{\circ}$. In addition, the parsec-scale jet of Mrk501 exhibits a transverse RM gradient [12, 13], with the larger-magnitude RM values present on the side of the jet with a lower degree of polarization, as is expected for a helical jet B field. Although such results are available for only one or two sources, this approach could potentially be quite valuable when applied to a larger number of AGN of different types. Profile fitting can also be used to investigate change in the pitch angle and viewing angle of the helical field along the jet.

Although it can be somewhat model-dependent, fitting such as that carried out by Murphy et al. [27] can also provide a useful new tool for deriving very fundamental information about the jets, since this fitting yields the viewing angle of the jet in the jet rest frame, $\theta^{\prime}$. Using this angle, the measured superluminal speed $\beta_{a p p}$, and the formulas

$$
\begin{aligned}
\sin \theta^{\prime} & =\frac{\sin \theta}{\Gamma[1-\beta \cos \theta]} \\
\beta_{a p p} & =\frac{\beta \sin \theta}{1-\beta \cos \theta}
\end{aligned}
$$

where $\theta$ is the viewing angle of the jet in the observer rest frame, $\beta$ is the speed of the jet divided by the speed of light and $\Gamma$ is the Lorentz factor of the jet, it is possible to solve for the jet velocity $\beta$ and the viewing angle $\theta$. Such an analysis for Mrk501 yielded $\beta=0.96$ and $\theta=15^{\circ}$ 


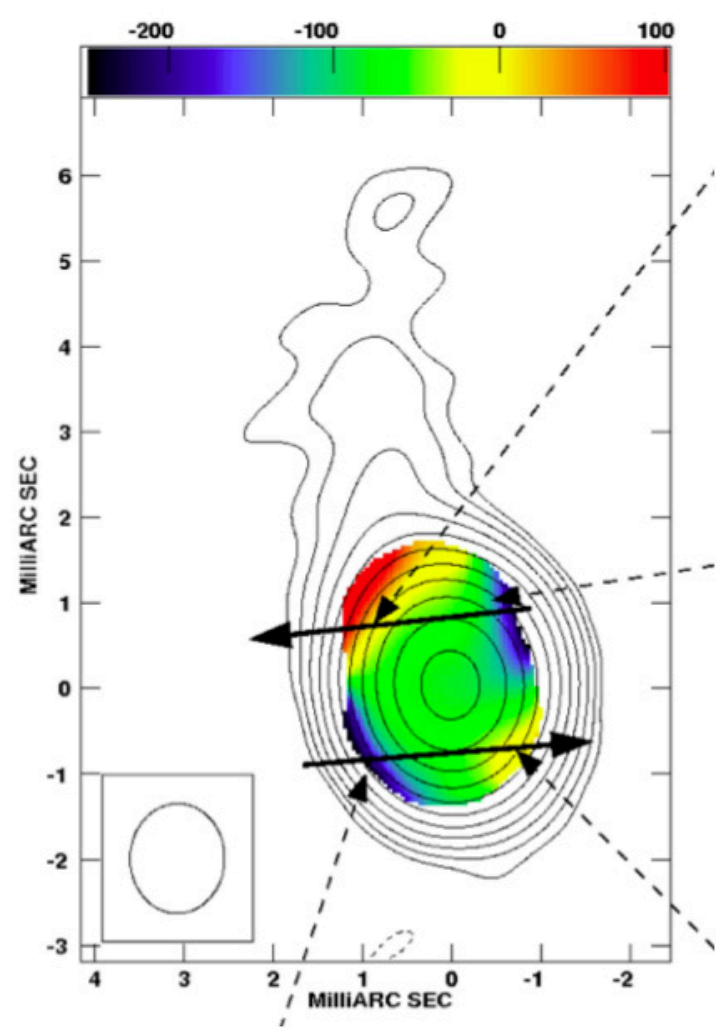

Figure 6. Example of a reversal of an observed transverse RM gradient with distance along the jet (results of Mahmud et al. [26]).

[27], consistent with but more constraining than the earlier results of Giroletti et al. [16] [ $\left.\beta \geq 0.88, \theta \leq 27^{\circ}\right]$.

A surprising result to emerge from recent work on transverse Faraday rotation gradients are recent observations of reversals of transverse RM gradients both in time [25] and with distance from the jet base [26]; Fig. 6). Although this result at first seems mysterious, it can plausibly be explained if the "outgoing" B field in the inner accretion disk closes in the outer accretion disk, giving rise to a "nested helical field" structure for the overall jet B field (Fig. 7). The total Faraday rotation integral must be taken through both the inner and outer regions of helical field; if the directions of the azimuthal field components in the inner and outer helical-field regions are opposite, they will tend give rise to RM gradients in opposite directions across the jet. The net observed RM gradient will be determined by whichever of the two regions dominates the overall observed Faraday rotation. Thus, these new observations provide the first direct evidence for the presence of "return fields" in more extended regions surrounding AGN jets. Since this interpretation requires that the azimuthal field component has opposite directions in the inner and outer regions of helical field, this can potentially place important constraints on magnetic-field models for AGN jets.

There have also been some reports of transverse Faraday-rotation gradients on kiloparsec scales (Kronberg et al. [20], Gabuzda et al. [14], Algaba et al. [2]), although these have not been analyzed for reliability as thoroughly as those investigated on parsec scales. Nevertheless, a number of the identified RM gradients appear to be significant, demonstrating that a toroidal or helical field component can also be present on kiloparsec scales. Kiloparsecscale transverse RM gradients appear to be considerably fewer in number, apparently reflecting an increased contribution from a random (turbulent) field component on these scales.

\section{Monte Carlo Simulations Investigating Image Properties}

As is indicated above, a number of studies carried out in different groups around the world had reported detections of transverse Faraday-rotation gradients across AGN jets on parsec scales. Further, the theoretical simulations of the propagation of jets from a rotating black hole/accretion disk system carried out by Broderick \& McKinney [6] clearly showed the presence of transverse RM gradients, and also showed that these could sometimes remain visible even when convolved with a beam that was much larger than the intrinsic jet width (e.g. a 0.9-mas beam and an intrinsic jet width at its base of about 0.05 mas; Fig. 8, right). Convolution with a beam larger than the jet led to non-monotonic behaviour of the gradients in the core region in some, but not all, cases. In fact, comparing the bottom two panels of Fig. 8 of [6] [see Fig. 8 in the current paper], convolution with beams considerably larger than the intrinsic jet size led to smoother behaviour of the gradients than convolution with beams that were comparable to or only slightly larger than the intrinsic jet width. Thus, overall, these theoretical simulations suggested that it should, in principle, be possible to detect the presence of transverse RM gradients across parsec scale jets, including across the core regions, although the magnitudes of these gradients would of course be severely depressed due to convolution with the relatively large VLBI beam.

At roughly the same time, Taylor \& Zavala [34] proposed four criteria for the reliability of transverse RM gradients, requiring that: (1) the gradients span at least three "resolution elements" across the jet, (2) the difference in the RM be at least three times the typical error in the RM, (3) the gradient be in an optically thin region and (4) the gradient be monotonic and smooth (within the errors). In fact, the second and fourth of these criteria had been applied in essentially all previous studies of transverse RM gradients, although there was some lack of clarity about the most correct way to estimate the uncertainties on the $\mathrm{RM}$ values at specified locations in an RM image. The first and third were presented without any firm justiication or demonstration using simulated data, but apparently appeared plausible to many researchers, and so were often adopted without any clear demonstration of their correctness.

It was particularly urgent to clarify the situation with regard to the first criterion, interpreted as requiring that an RM gradient span three beamwidths, since this would have 

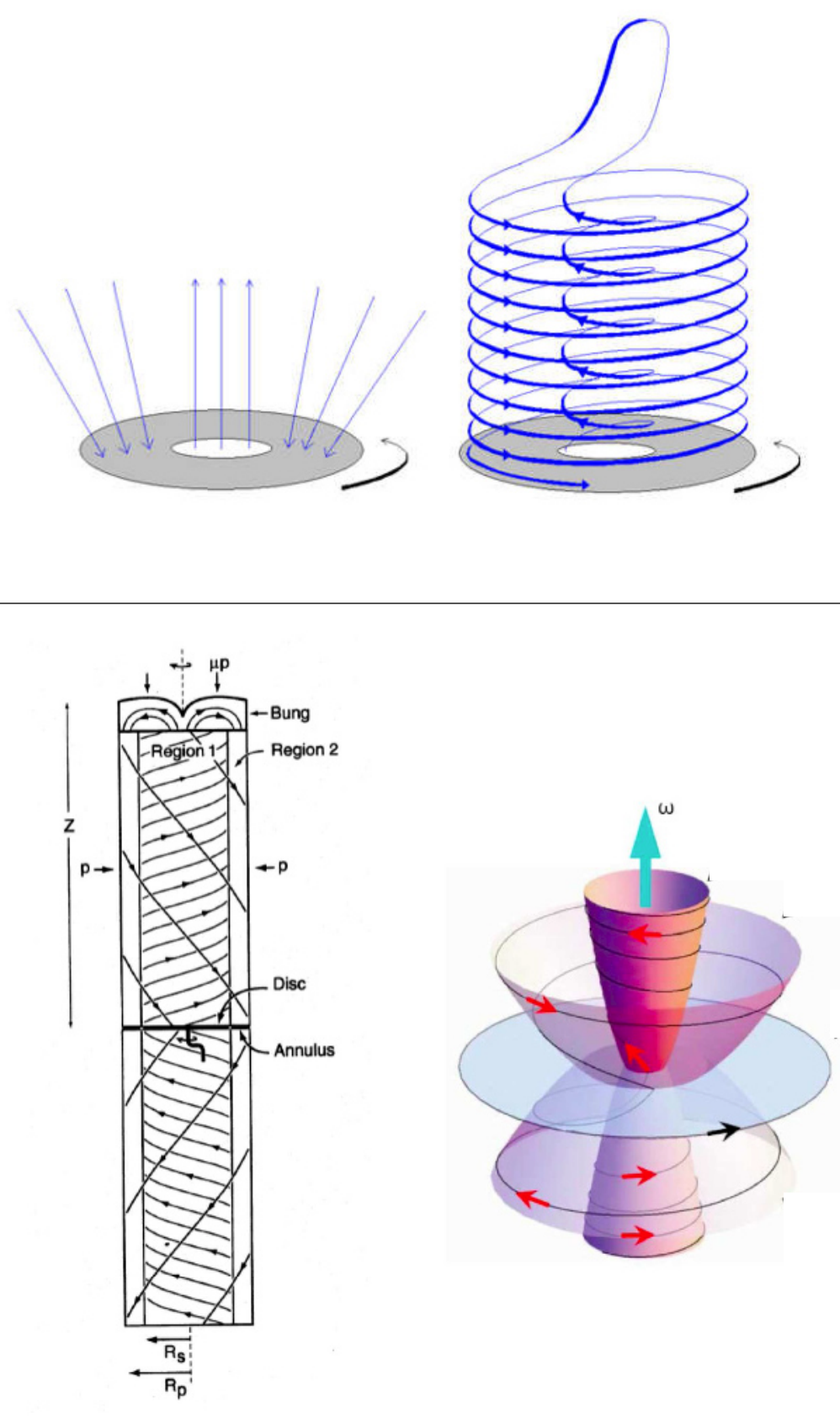

Figure 7. Top: Schematic illustrating how the winding up of the outgoing (inner accretion disk) and return (outer accretion disk) fields can give rise to a "nested helical field" structure (taken from [24]). The net Faraday rotation observed for the jet will depend on the relative contributions of both regions of helical field. Bottom: Two different outgoing and return field configurations, in which the two regions have azimuthal field in the same direction (left; taken from [23]) and in opposite directions (right; taken from [9]). The former cannot give rise to reversals of the direction of the net RM gradient, but the latter can. 

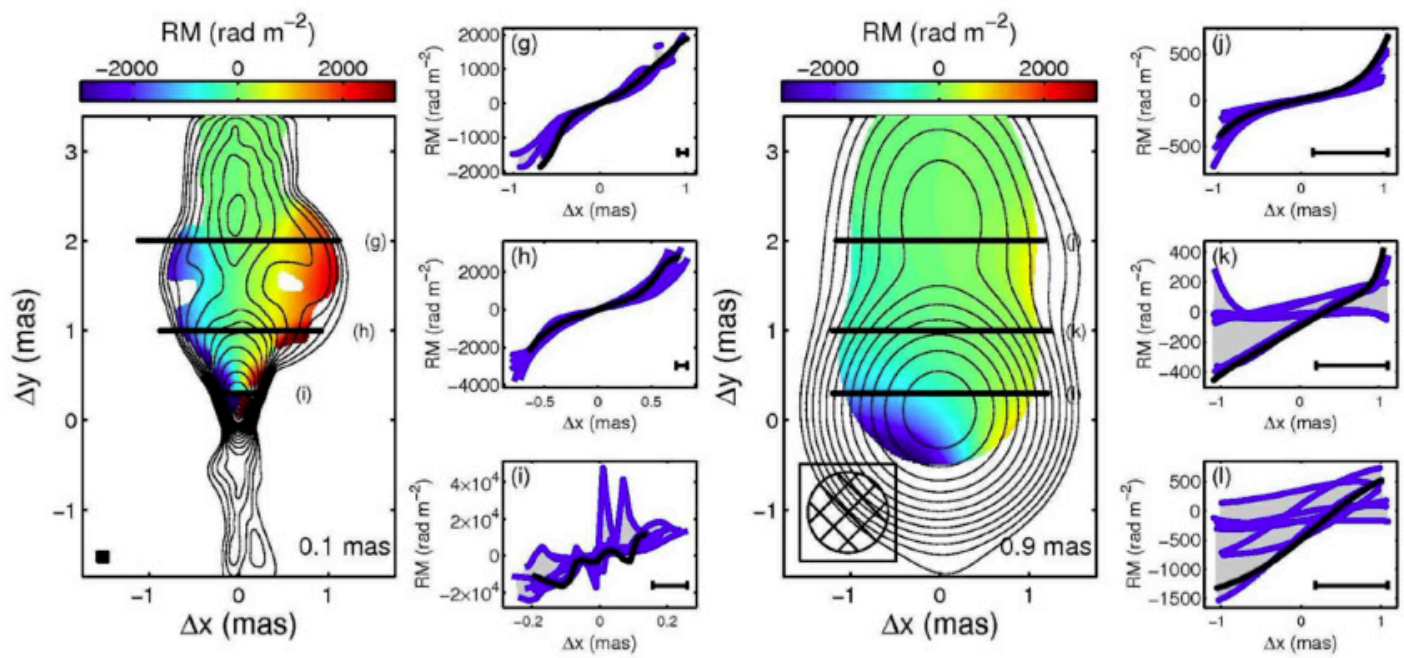

Figure 8. Simulated RM maps of Broderick \& McKinney [6], convolved with beams that are about a factor of two larger than the intrinsic jet width at the jet base (left) and about a factor of 15-20 larger than the intrinsic jet width at the jet base (right). The plots to the right of each RM map show transverse RM profiles for the slices shown across the jets in black for a variety of azimuthal angles.

rejected nearly all transverse RM gradients previously reported in the literature.

A first important step was taken by Hovatta et al. [18], who carried out Monte Carlo simulations based on realistic "snapshot" baseline coverage for VLBA observations at 7.9, 8.4, 12.9 and $15.4 \mathrm{GHz}$ aimed at investigating the statistical occurrence of spurious RM gradients across jets with intrinsically constant polarization profiles. Inspection of the right-hand panel of Fig. 30 of [18] [see Fig. 9] shows that the fraction of spurious $3 \sigma$ gradients was no more than about $1 \%$, even for the smallest observed RMgradient widths they considered, about 1.4 beamwidths. They also found relatively few $2 \sigma$ gradients, although this number reached about $7 \%$ for observed jet widths of about 1.5 beamwidths. These results thus gave the first clear indication that the three-beamwidth criterion of [34] was too severe (note that Hovatta et al. [18] were fairly conservative in interpreting their own results).

The results of Hovatta et al. [18] have also now been confirmed by similar Monte Carlo simulations carried out by Algaba [1] for simulated data at 12,15 and $22 \mathrm{GHz}$ and by Murphy \& Gabuzda [28] for simulated data at 1.38, $1.43,1.49$ and $1.67 \mathrm{GHz}$ and at the same frequencies as those considered in [18]. The general behaviour exhibited in these three sets of simulations is similar, although details depend on the frequency range considered. The sensitivity of the $1.38-1.67 \mathrm{GHz}$ frequency range used for the simulations of [28] yielded a negligible number of spurious $3 \sigma$ gradients and fewer than $1 \%$ spurious $2 \sigma$ gradients, even for observed jet widths of only 1 beamwidth. Thus, all these Monte Carlo simulation results demonstrate that the three-beamwidth criterion of [34] is too severe (see [28] for more detail).

Another key outcome of the Monte Carlo simulations of Hovatta et al. [18] is an empirical formula that can be used to estimate the uncertainties in intensity (Stokes $I$, $Q$ or $U$ ) images, including the uncertainty due to resid-

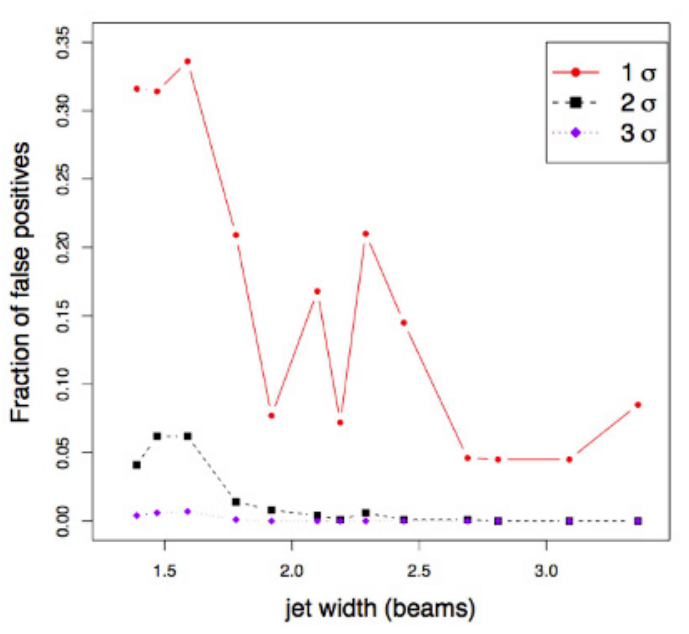

Figure 9. Results of Hovatta et al. [18] for the occurrence of spurious RM gradients with various significance levels and spanning various widths.

ual instrumental polarization ("D-terms") that has been incompletely removed from the visibility data. In regions of source emission where the contribution of the residual instrumental polarization is negligible, the typical uncertainties in individual pixels are approximately 1.8 times the rms deviations of the flux about its mean value far from regions of source emission. This is roughly a factor of two greater than the uncertainties that have usually been assigned to the intensities measured in individual pixels in past studies, indicating that the past uncertainties have been somewhat underestimated.

Two more sets of Monte Carlo simulations based on realistic snapshot VLBA baseline coverage carried out recently are worthy of mention here. These adopted a different approach to those described above: instead of con- 

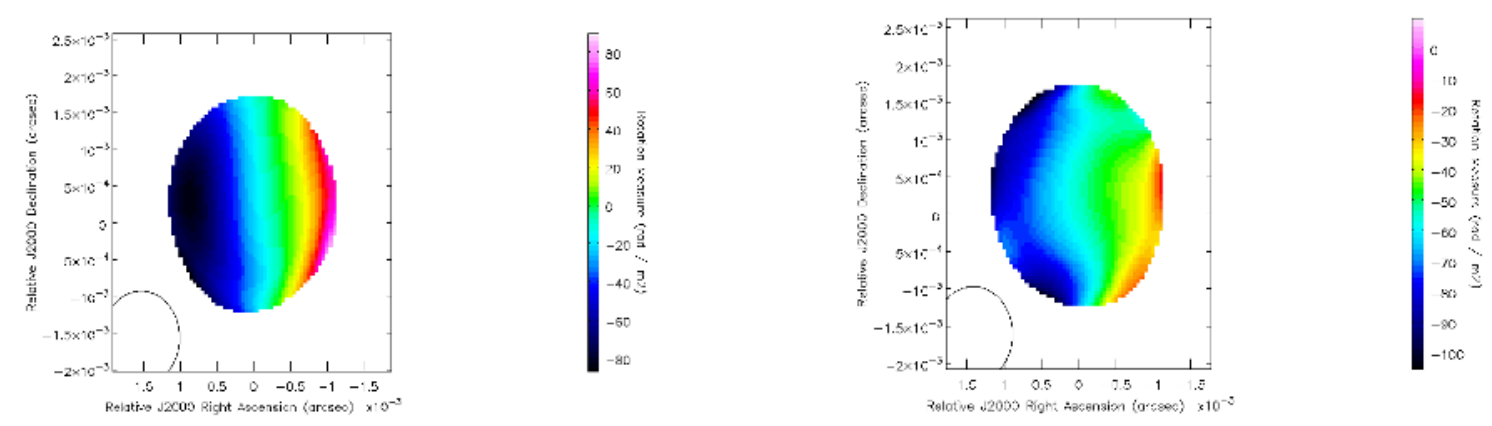

Figure 10. Simulated RM maps of Mahmud et al. [26] showing the ability to detect transverse RM gradients even when the intrinsic jet width is much smaller than the beam width. The intrinsic jet widths are $1 / 10$ (left) and 1/20 (right) of a beamwidth; the direction of the jet is straight upward in both images, so that the observed gradients are transverse to the jet. For more examples, see [28].

sidering the occurrance of spurious RM gradients across jets with constant polarization, they considered simulated jets with various widths and with transverse RM gradients of various strengths, convolved with various size beams. Mahmud et al. [26] carried out such simulations for 4.6, 5.0, 7.9, 8.4, 12.9 and 15.4 GHz VLBA data (Fig. 10), while Murphy \& Gabuzda [28] considered simulations for $1.38,1.43,1.49$ and $1.67 \mathrm{GHz}$. These simulations clearly showed that, with realistic noise and baseline coverage, the simulated RM gradients could remain clearly visible, even when the jet width was as small as $1 / 20$ of a beam width. In the study by Murphy \& Gabuzda [28], at least $98 \%$ of simulated RM maps with an intrinsic polarization of $15 \%$ and an intrinsic RM range of +30 to $-30 \mathrm{rad} / \mathrm{m}^{2}$ showed their transverse RM gradients at a level $\geq 3 \sigma$ when the intrinsic jet width was at least $1 / 5$ of a beamwidth. These simulations clearly demonstrate that the width spanned by an RM gradient is not a key criterion for its reliability.

The question of the reliability of transverse RM gradients observed across partially optically thick core regions remains somewhat open, and further simulations are required to investigate this. Bearing in mind, however, that what we observe as the "VLBI core" with beamwidths of the order of a milliarcsecond is actually a blend of the optically thick base of the jet and optically thin regions in the inner jet, it is likely in many cases that the polarization of the observed core components is dominated by optically thin emission. This is consistent with the fact that the observed spectral indices near the map peak are often fairly flat, rather than being strongly positive. It is also important to note that the optical depth at which the $90^{\circ}$ rotation in polarization angle in the transition from optically thick to optically thin occurs is appreciably greater than $\tau=1$ (e.g. Cawthorne \& Hughes [7]). In addition, the theoretial simulations of Broderick \& McKinney [6] show smooth, monotonic RM gradients across the observed VLBI core when they are convolved with a 0.9 -mas beam (see Fig. 8). These various facts provide "circumstantial evidence" that transverse RM gradients across core regions may often be reliable, but further simulations are required to test this more directly.

\section{Conclusion}

A variety of image-alignment techniques have how been developed and are being actively applied, both in studies of individual sources (e.g. O'Sullivan \& Gabuzda [29]) and in analyses of data for larger samples (e.g. Pushkarev et al. [30]). Core-shift and core B-field studies have now been carried out for relatively large numbers of frequencies and large source samples for the first time. Most of the results of these analyses are consistent with equipartition in the core region; suggesting that $B \sim r^{-1}$ [29]. The $15-\mathrm{GHz}$ core $B$ fields range from about $0.02 \mathrm{G}$ to about $0.8 \mathrm{G}$; there is evidence that the core B fields are somewhat lower in BL Lac objects than in quasars [30].

Various parsec-scale Faraday-rotation studies (e.g. $[18,36,37])$ have shown that the core Faraday rotation measures (RMs) are usually substantially higher than the jet RMs, presumably due to the presence of higher electron densities and higher B fields on smaller scales in the sources. The core RMs are somewhat lower in BL Lac objects than in quasars. Transverse RM gradients and transverse polarization structure provide direct evidence for helical jet B fields, which would naturally be formed by the rotation of the central black hole and its accretion disk combined with the jet outflow. The observation of reversals of transverse RM gradients both in time [25] and with distance from the jet base [26] can be explained if the jet has a "nested helical magnetic field" structure with the directions of the azimuthal field components in the inner and outer regions of helical field being opposite. These observations thus provide the first evidence for the existence of a return field in the region surrounding the jet.

Monte Carlo simulations of transverse RM gradients carried out by Hovatta et al. [18], Algaba [1], Mahmud et al. [26] and Murphy \& Gabuzda [28] have demonstrated that the "three-beamwidth" criterion of Taylor \& Zavala [34] for the reliability of transverse RM gradients is too severe. The best criteria for the reliability of transverse RM gradients are that the total RM difference spanned be $>3 \sigma$, and that the gradients be monotonic within the uncertainties. Somewhat non-intuitively, simulated RM images with transverse gradients have shown that these gradients can be detectable even when the intrinsic jet width 
is substantially less than the beam width (as little as $1 / 20$ of a beamwidth!). We should note here that these results refer to the ability to detect the presence of transverse RM gradients; reliably deriving source parameters based on measurements of transverse RM gradients will require appreciably better resolution. The usual practice of assigning intensity (Stokes $I, Q$ or $U$ ) errors to be equal to the rms flux deviations in the corresponding image far from regions of source emission appreciably underestimates the real uncertainties (by at least a factor of about 1.8) [18].

Important future work will include studies to reevaluate the significance of previously reported results (e.g. for RM gradients) based on improved approaches to estimating uncertainties. Studies aimed at further improving our understanding of the uncertainties in fluxes measured in individual pixels and the correlations between the fluxes measured in nearby pixels, will also be important. The development of alternative imaging techniques for VLBI data, such as maximum-entropy polarization imaging (e.g. Coughlan \& Gabuzda [10]) and RM synthesis will also play a key role. Future polarization space-VLBI observations at centimeter wavelengths hold a great deal of promise for furthering our understanding of AGN jets, since they can provide high sensitivity to phenomena such as Faraday rotation or low-frequency absorption while also providing high angular resolution.

\section{References}

[1] Algaba J. C., MNRAS 429, 3551-3563 (2013)

[2] Algaba J. C., Asada K. \& Nakamura M., ApJ Letters, submitted (2013)

[3] Asada K., Nakamura M., Inoue M., Kameno S. \& Nagai H., ApJ 720, 41-45 (2010)

[4] Blandford R. D., Astrophysics and Space Science Library 103, 15-33 (1993)

[5] Blandford R. D. \& Königl A., ApJ 232, 34-48 (1979)

[6] Broderick A. E. \& McKinney J. C., ApJ 725, 750-773 (2010)

[7] Cawthorne T. V. \& Hughes P. A., ApJ 771, 60-70 (2013)

[8] Cohen M. H., Cannon W., Purcell G. H., Shaffer D. B., Broderick J. J., Kellermann K. I., \& Jauncey D. L., ApJ 170, 207-217 (1971)

[9] Contopoulos I., Christodoulou D. M., Kazanas D. \& Gabuzda D. C., ApJ Let 702, 148-152 (2009)

[10] Coughlan C. P. \& Gabuzda, The Innermost Regions of Relativistic Jets and their Magnetic Fields (2013)

[11] Croke S. M. \& Gabuzda D. C., MNRAS 386, 619626 (2008)

[12] Croke S. M., O'Sullivan S. P., and Gabuzda D. C., MNRAS 402, 259-270 (2010)
[13] Gabuzda D. C., Murray É. \& Cronin P., MNRAS Letters 351, 89-93 (2004)

[14] Gabuzda D. C., Christodoulou D., Contopoulos I. \& Kazanas D., Journal of Physics: Conference Series 355 (2012)

[15] Gabuzda D. C., Cantwell T. M. \& Cawthorne T. V., MNRAS in press (2013)

[16] Giroletti M., Giovannini G., Feretti L., Cotton W. D., Edwards P. G., Lara L., Marscher A. P., Mattox J. R., Piner B. G. \& Venturi T., ApJ 600, 127-140 (2004)

[17] Gómez J. L., Roca-Sogorb M., Agudo I., Marscher A. P. \& Jorstad S. G., ApJ 733, 11-23 (2011)

[18] Hovatta T., Lister M. L., Aller M. F., Aller H. D., Homan D. C., Kovalev Y. Y., Pushkarev A. B. \& Savolainen T., AJ 144, 105-139 (2012)

[19] Kovalev Y. Y., Lobanov A. P., Pushkarev A. B. \& Zensus J. A., A\& A 483i, 759-768 (2008)

[20] Kronberg P. P., Lovelace R. V. E., Lapenda G. \& Colgate S. A., ApJ Letters 741, L15-L19 (2011)

[21] Lister M. L. \& Homan D. C., AJ 130, 1389-1417 (2005)

[22] Lobanov A. P., A\& A 330, 79-89 (1998)

[23] Lynden-Bell D., MNRAS 279, 389-401 (1996)

[24] Mahmud M., PhD Thesis, University College Cork (2010)

[25] Mahmud M., Gabuzda D. C., and Bezrukovs V. I., MNRAS 400, 2-12 (2009)

[26] Mahmud M., Coughlan C. P., Murphy E., Gabuzda D. C. \& Hallahan D. R., MNRAS 431, 695-709 (2013)

[27] Murphy E., Cawthorne T. V., and Gabuzda D. C., MNRAS 430, 1504-1515 (2013)

[28] Murphy E. \& Gabuzda, The Innermost Regions of Relativistic Jets and their Magnetic Fields (2013)

[29] O’Sullivan S. P. \& Gabuzda D. C., MNRAS 400, 2642 (2009)

[30] Pushkarev A. B., Hovatta T., Kovalev Y. Y., Lister M. L., Lobanov A. P., Savolainen T. \& Zensus J. A., A\& A 545, 113-128 (2012)

[31] Sokolovsky K. V., Kovalev Y. Y., Pushkarev A. B. \& Lobanov A. P., A\& A 532, 38-69 (2011)

[32] Taylor G. B., ApJ 506, 637-646 (1998)

[33] Taylor G. B., ApJ 533, 95-105 (2000)

[34] Taylor G. B. \& Zavala R. T., ApJ Letters 722, 183187 (2010)

[35] Walker R. C., Dhawan V., Romney J. D., Kellermann K. I. \& Vermeulen R. C., ApJ 530, 233-244 (2000)

[36] Zavala R. T. \& Taylor G. B., ApJ 589, 126-146 (2003)

[37] Zavala R. T. \& Taylor G. B., ApJ 612, 749-779 (2004) 